Buckland SS, Homer CSE. (2007) Estimating blood loss after birth: Using simulated clinical examples, Women and Birth 20(2): 85-88.

\title{
Estimating blood loss after birth: Using simulated clinical examples
}

\begin{abstract}
Aim: To determine the accuracy of the estimation of blood loss using simulated clinical examples.
\end{abstract}

Setting: Over 100 attendees came together at a seminar about postpartum haemorrhage in June 2006. Five blood loss assessment stations were constructed, each containing a simulated clinical example. Each station was numbered and was made up of a variety of equipment used in birthing suites. Over five litres of 'artificial' blood was made. The artificial blood was similar to the colour and consistency of real blood.

Sample: A convenience sample of 88 participants was given a response sheet and asked to estimate blood loss at each station. Participants included midwives, student midwives and an obstetrician.

Results: Blood in a container (bedpan, kidney dish) was more accurately estimated than blood on sanitary pads, sheets or clothing. Lower volumes of blood were also estimated correctly by more participants than the higher volumes.

Discussion: Improvements are still needed in visual estimation of blood loss following childbirth. Education programs may increase the level of accuracy.

Conclusion: We encourage other clinicians and educators to embark upon a similar exercise to assist midwives and others to improve their visual estimation of blood loss after birth. Accurate estimations can ensure that women who experience significant blood loss can receive appropriate care and the published rates of postpartum haemorrhage are correct.

Keywords: Midwifery; Uterine haemorrhage; Postnatal period; Labour, Obstetrics; Education 


\section{Introduction}

There are major complications for women related to blood loss during pregnancy, labour and birth and in the postnatal period. Obstetric haemorrhage remains a significant cause of maternal mortality and morbidity, even in developed countries. ${ }^{1}$ In Australia, the most common cause of direct maternal death in the triennium 19971999 was obstetric haemorrhage (eight deaths) compared with five from the previous triennium. ${ }^{2}$ The report, Maternal Deaths in Australia 1997-1999 stated that "this category continues to be of concern and may be increasing" (page xiv) Similarly in the United Kingdom (UK), the number of deaths resulting from haemorrhage has more than doubled from seven in 1997-1999 to 17 in 2000-2002. ${ }^{1}$ Major maternal morbidity related to haemorrhage, in particular, postpartum haemorrhage (PPH), includes iron deficiency anemia, prolonged hospital stay, delay or failure of lactation due to pituitary effects, exposure to blood products, acute renal failure, the need for surgical intervention and in cases of intractable $\mathrm{PPH}$, the need for hysterectomy. ${ }^{34}$

One of the major recommendations from the most recent Why Mothers Die Report in the United Kingdom was related to the accurate quantification of blood loss. ${ }^{1}$ Quantification assists with the restoration of blood volume and guides decisions around the use of transfusions of crystalloids, red cells and fresh frozen plasma. ${ }^{5}$ For example, the infusion of crystalloids (normal saline or Hartman's), in a volume at least three times the measured volume lost, is suggested to restore circulating volume. ${ }^{6}$ Red cell transfusions are recommended when $30-40 \%$ of blood volume is lost. $^{7}$

Accurate measurement of blood loss is nonetheless challenging, particularly in the practice setting. A recent observational study, using clinical reconstructions, demonstrated significant underestimation of actual blood loss in five of twelve clinical scenarios with no overestimations. ${ }^{8}$ Previously in Australia, research showed that midwives and other health professionals underestimated blood loss after birth by 30-50\%. ${ }^{9}$ Quantifying blood loss is not only important for immediate management, it is also necessary to measure the incidence of haemorrhage such as $\mathrm{PPH}$ and to monitor trends. It is recognised that the incidence of PPH may be underestimated by up to $50 \%$, due to the clinical difficulty in accurately estimating blood loss. ${ }^{10}$

Assessment of haemodynamic status requires a combination of approaches. Visual estimation is one method of assessment that should be used as a means to guide management. A drop in the woman's haematocrit of greater than 10 points can also guide diagnosis of significant haemorrhage, although only after the event. Clinical evaluation of the woman (colour, dizziness, level of consciousness) and cardiovascular assessments, including heart rate, blood pressure and cardiac output, will also assist the decision making process. However, silent ischaemia may occur in the presence of stable vital signs. ${ }^{5}$ Compensatory changes may sustain a women's circulatory status at near normal levels, despite large blood loss, until a critical level is reached and there is a sudden and profound change in blood pressure and pulse to indicate shock. ${ }^{11}$ Women can lose $1000-1500 \mathrm{~mL}$ and show only a slight fall in blood pressure $(80-100 \mathrm{mmHg}$ ) and up to $2000 \mathrm{~mL}$ before a marked fall $(70-80 \mathrm{mmHg})$ in blood pressure is seen. ${ }^{10}$ In women with marked anaemia, much less blood loss can still result in marked changes in haemodynamic status. Clearly, a combination of approaches is needed. While clinical assessment is important, visual, accurate visual 
estimation of blood loss also plays is significant role in the management of haemorrhage.

The aim of the exercise described in this paper was to determine the accuracy of visual estimation of blood loss using simulated clinical examples. This paper reports the selection and construction of five simulated clinical examples and the results obtained from 88 volunteers including midwives, student midwives and an obstetrician at a seminar about postpartum haemorrhage.

\section{Method}

Simulated clinical examples (Table 1) were constructed at five stations using previous Australian research as a guide. ${ }^{9}$ Each station was numbered and was made up of a variety of equipment similar to those used in birthing suites. One of the stations used a kidney dish which had visible measurements on the inside. None of the other stations used equipment with measurements.

$<$ Insert Table 1 here $>$

Over five litres of 'artificial' blood was made for the stations. The artificial blood recipe is provided in Figure $1^{12}$. The artificial blood was close to the same colour and consistency as real blood and no other fluid was mixed with it at any of the stations.

$<$ Insert Figure 1 here $>$

A convenience sample was used. Over 100 midwives, student midwives and obstetricians came together at a seminar about postpartum haemorrhage in June 2006. A diverse combination of presenters from government, non-government organisations, and various hospitals including midwives, obstetricians and researchers explored postpartum haemorrhage and the care provided to women in such an emergency. During the morning, five sessions were presented, including a paper reporting the PPH rates in New South Wales, Australia; the different approaches to the management of the third stage of labour; a consumer's view after experiencing a $\mathrm{PPH}$; and, a presentation about the challenges of accurately estimating blood loss after birth. Following the morning session, all attendees were invited to participate after an explanation of the exercise was provided.

Participants were assured of confidentiality and anonymity, were aware that the results would be used to assess the accuracy of estimations and may be published in the future. Written consent to participate was not sought - participation was taken as consent. Of the 100 delegates at the seminar, 88 volunteered to participate including 82 midwives, 1 obstetrician, and 5 midwifery students. Participants were given a preprinted response sheet and asked to estimate the actual loss at each station. Gloves were available so they could also touch the simulations.

The data from the response sheets were collected and entered onto a Microsoft Excel spreadsheet. The proportions of the participants who were exactly correct or within $50 \mathrm{mLs}(+/-)$ of the actual amount were calculated. The proportion of participants who under-estimated (less that $50 \mathrm{~mL}$ of the correct amount) or over-estimated (greater 
than $50 \mathrm{~mL}$ of the correct amount) the amount of blood in each example was then calculated.

The median, interquartile range and volume of error were calculated for each station in a manner similar to the most recent study of estimation of blood loss ${ }^{8}$ that was conducted in the UK. Median and interquartile ranges were used as the data were not normally distributed. The interquartile range is the difference between the third and first quartiles, that is, the distance between the 75th percentile and the 25th percentile and is a measure of statistical dispersion or spread. ${ }^{13}$ The interquartile range is a more stable statistic than the range, and is often preferred to that statistic. The interquartile ranges is essentially the range of the middle $50 \%$ of the data and as it uses the middle $50 \%$, it is not affected by outliers or extreme values.

\section{Results}

Blood in a container (bedpan, kidney dish) was more accurately estimated than blood on sanitary pads, sheets or clothing. Lower volumes of blood were also estimated correctly by more participants than the higher volumes.

At $350 \mathrm{~mL}$, where the blood was contained in a bedpan, almost half (49\%) of participants were either exactly accurate or within $50 \mathrm{~mL}$ of the actual amount. At one of the $600 \mathrm{~mL}$ stations, where blood was contained in a kidney dish, $41 \%$ of participants were exactly accurate or within $50 \mathrm{~mL}$. In the other $600 \mathrm{~mL}$ station, which used sanitary pads and pyjama pants, only $4 \%$ of participants were exactly accurate or within $50 \mathrm{~mL}$. At $800 \mathrm{~mL}$, where blood stained blue absorbent sheets were used, only $16 \%$ of participants were exactly accurate or within $50 \mathrm{~mL}$. Finally, at the highest volume, $1000 \mathrm{~mL}$, where the blood was on a sheet and a plastic mackintosh, only $4 \%$ of participants were accurate in their estimation or within 50mL (Figure 2).

$<$ Insert Figure 2 here $>$

The median estimated blood loss was similar to the actual blood volume at the lower levels $(350 \mathrm{~mL})$ and when a container was used $(600 \mathrm{~mL})$. However, once clothing, linen or sanitary pads were blood stained, the accuracy decreased (Table 3).

\section{$<$ Insert Table 2 here $>$}

As a group, only one participant accurately estimated the estimated blood loss at three stations, eight participants accurately estimated two stations, 26 participants accurately estimated only one station and 52 did not estimate any correctly.

\section{Discussion}

Visual estimation of blood loss after childbirth is undertaken often by midwives and doctors working in maternity settings and anecdotally seems to lack accuracy. The process has also been shown to be inaccurate in a number of studies ${ }^{8914}$ and therefore, it could be suggested that it lacks clinical usefulness.

In this exercise, participants were more accurate in their estimations of blood loss when the blood was both a smaller amount and contained within a bowl or kidney dish, especially if measurements were visible, as the case with the kidney dish. 
Clearly, estimation is more difficult when the blood is soaked into clothing, linen or sanitary pads. The difference in the median estimated blood loss at the two $600 \mathrm{~mL}$ volumes is considerably different $(+50 \mathrm{~mL}$ and $+400 \mathrm{~mL})$. The former station contained the blood within a kidney dish while the latter included blood soaked clothing and sanitary pads. The difference indicates the difficulty of visual estimation in the absence of a container or an ability to weigh the items. The overestimation may also be related to the education that participants had received at the seminar they were attending where the problem of underestimation was highlighted.

The previous Australian study had a sample of 21 midwives and 5 medical officers. ${ }^{9}$ Our exercise used a similar process with a convenience sample of 87 midwives and students and one obstetrician who were attending a dedicated day on PPH. The level of underestimation may be lower than could be expected as participants were attending a seminar on PPH. One of the sessions prior to undertaking the estimation was about the problems relating to visual estimation including the data from the previous Australian study. ${ }^{9}$ It is possible that the rates of underestimation may have been higher had this education not been undertaken. Nonetheless, it is evident that maternity care providers still underestimate blood loss, especially at the higher volumes.

Previous studies have examined whether education could improve visual estimations. One study in the United States showed that the estimations made by obstetric nurses who worked in labour and birth units improved after education and the introduction of a standard approach to measurement. ${ }^{15}$ A more recent study, again in the United States but this time with doctors and medical students, demonstrated significant improvements in the estimation of blood loss after a 20 minute educational sesison. ${ }^{14}$

This exercise was limited as it was conducted in an unrealistic clinical setting (at a seminar) and used 'artificial' blood. It is difficult to have a large sample of midwives in a real clinical setting unless it is a very large unit. Equally, occupational health and safety concerns meant that real blood could not be used. Even though the blood was 'artificial', the participants remarked on how realistic it looked, especially the clots. We also did not collect any demographic data on the participants so we cannot analyse the estimations by years of experience, place of work or practice setting. This was undertaken as a 'fun' exercise and we were keen to engage as many of the attendees as possible. We were concerned that if we required too much additional information, attendees may be reluctant to participate. Being an exercise at a seminar is also a limitation. Participants may have been more likely to be offhand and frivolous about their estimations as it was an 'activity' at a seminar and not real practice. The results may be different if the exercise were repeated within a real clinical setting, for example, a labour ward, as clinicians may put more gravidity on the situations and take more care with estimations. In addition, we did not collect information on the usual work settings of the participants. It could be suggested that many worked in settings where estimating blood loss was usual in practice, as the seminar they attended was on PPH, however this cannot be assumed. Participants who regularly estimated blood loss in labour and birth settings may have been more accurate than those who worked in other areas. Future research could examine whether role and work setting play a role in accuracy. 


\section{Conclusion}

The findings of this exercise support recommendations for maternity care providers to improve their skills of estimating blood loss. Midwives and doctors need ongoing information about estimation of blood loss in clinical situations.

We encourage other clinicians and educators to embark upon similar exercises to assist midwives and others to improve their visual estimation of blood loss after birth and to consider the benefit of education to improve estimation. Accurate estimations can ensure that women who experience significant blood loss can receive appropriate care and the published rates of PPH are correct. 


\section{References}

1. Confidential Enquiry into Maternal and Child Health. Why mothers die 2000-2004. The Sixth Report of the Confidential Enquires into Maternal Deaths in the United Kingdom. Executive Summary and Key Findings. London: RCOG Press, 2004.

2. Slaytor E, Sullivan EA, King JF. Maternal Deaths in Australia 1997-1999. Canberra: Australian Institute of Health and Welfare, 2004.

3. Cameron CA, Roberts CL, Olive EC, Ford JB, Fischer WE. Trends in postpartum haemorrhage. Australian \& New Zealand Journal of Public Health 2006;30(2):151-6.

4. Anderson J, Etches D, Smith D. Postpartum hemorrhage: third stage emergency. Chapter J. In: Baxley E, Damos J, Eissinger S, editors. Advanced Life Support in Obstetrics (ALSO) Course Syllabus, . 4th ed. Kansas American Academy of Family Physicians, 2000.

5. Stainsby D, MacLennan S, Hamilton P. Management of massive blood loss: a template guideline. British Journal of Anaesthesia 2000;85(3):487-91.

6. World Health Organization. Managing Complications of Pregnancy and Childbirth: A Guide for MIdwives and Doctors. Geneva: Department of Reproductive Health and Research, World Health Organization, 2000.

7. American College of Surgeons. Advanced Trauma Life Support Course Manual. Ilinois: American College of Surgeons, 1997.

8. Bose P, Regan F, Paterson-Brown S. Improving the accuracy of estimated blood loss at obstetric haemorrhage using clinical reconstructions. BJOG: An International Journal of Obstetrics and Gynaecology 2006;113(8):919-924.

9. Glover P. Blood loss at delivery: How accurate is your estimation? . Australian Journal of Midwifery 2003;16(2):21-24.

10. Society of Obstetricians and Gynaecologists of Canada. Prevention and management of postpartum haemorrhage: SOGC Clinical Practice Guidelines No 88. Journal of Obstetrics and Gynaecology Canada 2000;22(4):271-81.

11. NSW Health. Postpartum Haemorrhage (PPH) - Framework for Prevention, Early Recognition and Management. Document Number PD2005_264. Sydney: NSW Health Department, 2005.

12. Anonymous. How to Make Fake Blood, unknown. http://www.dainter.com/infocus/fakeblood.htm. Accessed 10 June 2006

13. Everitt B. The Cambridge Dictionary of Statistics in the Medical Sciences. Cambridge: Cambridge University Press, 1995.

14. Dildy G, Paine A, George N, Velasco C. Estimating blood loss. Can teaching significantly improve visual estimation? Obstetrics \& Gynaecology 2004;104(1):30-6.

15. Luegenbiehl D. Improving visual estimations of blood volume on peripads. American Journal of Maternal/Child Nursing 1997;22(6):294-298. 